\title{
ARTICLE SHR-A1403, a novel c-Met antibody-drug conjugate, exerts encouraging anti-tumor activity in c-Met-overexpressing models
}

\author{
Chang-yong Yang ${ }^{1,2,3}$, Lei Wang ${ }^{4}$, Xing Sun ${ }^{3}$, Mi Tang ${ }^{3}$, Hai-tian Quan ${ }^{4}$, Lian-shan Zhang ${ }^{3}$, Li-guang Lou ${ }^{4}$ and Shao-hua Gou ${ }^{1,2}$
}

Emerging evidence demonstrates that a c-Met antibody-drug conjugate (ADC) has superior efficacy and safety profiles compared with those of currently available small molecules or antibody inhibitors for the treatment of c-Met-overexpressing cancers. Here we described both the in vitro and in vivo efficacies of SHR-A1403, a novel c-Met ADC composed of a humanized IgG2 monoclonal antibody against c-Met conjugated to a novel cytotoxic microtubule inhibitor. SHR-A1403 showed high affinity to c-Met proteins derived from human or monkey and potent inhibitory effects in cancer cell lines with high c-Met protein expression. In mice bearing tumors derived from cancer cell lines or patient HCC tissues with confirmed c-Met overexpression, SHR-A1403 showed excellent anti-tumor efficacy. Antibody binding with c-Met contributed to SHR-A1403 endocytosis; the subsequent translocation to lysosomes and cytotoxicity of the released toxin are speculated to be predominant mechanisms underlying the anti-tumor activity of SHR-A1403. In conclusion, SHR-A1403 showed significant anti-tumor activity in cancer cell lines, xenograft mouse models and an HCC PDX model, which all have high c-Met levels. These data provide references for SHR-A1403 as a potential therapy for the treatment of cancers with c-Met overexpression.

Keywords: SHR-A1403; c-Met ADC; anti-tumor; patient-derived xenograft (PDX); molecular mechanisms

Acta Pharmacologica Sinica (2019) 40:971-979; https://doi.org/10.1038/s41401-018-0198-0

\section{INTRODUCTION}

The receptor tyrosine kinase (RTK) c-Met (c-mesenchymal-epithelial transition factor) is the only known high-affinity receptor for hepatic growth factor (HGF), which is encoded by the protooncogene MET [1, 2]. Dysregulation of the HGF/c-Met pathway has been reported to promote tumor progression, angiogenesis, metastasis, and even resistance to certain therapies [1, 2]. Abundant evidence has shown that the aberrant activation of this pathway is a mechanism underlying the development of multiple cancers. Examples include MET exon 14 (METex14) mutations and MET amplification in non-small cell lung cancer (NSCLC) [3], MET amplification and protein overexpression in gastrointestinal cancers [4], and increased c-Met transcription and expression in hepatocellular carcinoma (HCC) [5]. These data indicate that the c-Met/HGF axis is a potential therapeutic target for multiple cancers.

Selective and non-selective broad-spectrum small molecule kinase inhibitors, which blunt downstream signaling transduction, and anti-c-Met antibodies, which interfere with ligand-receptor interactions, are currently available approaches for treating cancers with aberrantly activated c-Met signaling $[6,7]$. To date, only two non-selective broad-spectrum kinase inhibitors with c-Met inhibitory activity, crizotinib (Pfizer) and cabozantinib
(Exelixis), have been approved by the FDA for the treatment of NSCLC and medullary thyroid carcinoma, respectively $[8,9]$. The outcomes for many other small molecular selective kinase inhibitors in various stages of clinical trials, e.g., tivantinib [10], volitinib [11], and capmatinib [12], have been rather disappointing. Antibodies with normal structures targeting c-Met were reported to trigger c-Met dimerization and stimulate downstream signaling, which hampered their anti-tumor potency [13]. Thus, the "one-armed" anti-c-Met antibody onartuzumab [14] and the anti-HGF antibody rilotumumab [15], which lack agonistic activity, have shown greater promise. Disappointingly, limited clinical benefit was achieved from these potential therapies; studies revealed that the constitutive activation of this pathway through gene amplification, mutation, or HGF-dependent activation may be more predictive than c-Met protein overexpression alone $[16,17]$. Therefore, inappropriate screening strategies are additional factors that may influence these poor clinical outcomes.

In contrast, the antibody-drug conjugate (ADC) strategy, which only requires high protein expression for targeting and mediating the endocytosis of the conjugated toxins, could theoretically overcome the shortcomings of antibody-based therapies and enhance the targeting effects of small molecule inhibitors [18]. Because c-Met overexpression occurs in $30-50 \%$ of tumor types,

\footnotetext{
${ }^{1}$ Pharmaceutical Research Center and School of Chemistry and Chemical Engineering, Southeast University, Nanjing 211189, China; ${ }^{2}$ Jiangsu Province Hi-Tech Key Laboratory for

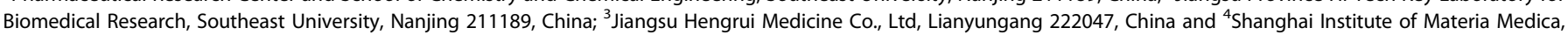
Chinese Academy of Sciences, Shanghai 201203, China

Correspondence: Shao-hua Gou (sgou@seu.edu.cn)

These authors contributed equally: Chang-yong Yang, Lei Wang
}

Received: 14 September 2018 Accepted: 22 November 2018

Published online: 14 January 2019 
including HCC, colorectal cancer, NSCLC, and gastroesophageal cancer, the application of ADC technology in c-Met-based therapies may improve clinical outcomes. Furthermore, tumor tissues have significantly higher c-Met expression compared with normal tissues, which should create a larger safety window for $A D C$ therapy. To our knowledge, the only clinical-stage (Phase 1) c-Met ADC is ABBV-399 from Abbvie, which is composed of an anti-c-Met antibody ABT-700 conjugated to a clinically applicable cytotoxic microtubule inhibitor MMAE (monomethylauristatin E) $[19,20]$. The preclinical data for ABBV-399 revealed robust antitumor efficacy, and recently disclosed Phase 1 data showed an acceptable safety profile and encouraging clinical benefit, especially in patients with c-Met overexpression [19, 20]. SHRA1403 is a novel, Phase 1 clinical-stage c-Met ADC consisting of a humanized anti-c-Met antibody (IgG2 subtype) conjugated via an uncleavable linker to a novel and improved version of a cytotoxic microtubule inhibitor (the free toxin) with the intention of overcoming the potential drawbacks associated with ABBV-399.

In this study, we investigated the preclinical in vitro and in vivo anti-tumor efficacy of SHR-A1403 and the underlying mechanisms to provide a scientific basis for clinical trials in patients with c-Met overexpression.

\section{MATERIALS AND METHODS}

Drugs and reagents

SHR-A1403 (ADC), SHR-A1403 mAb (the naked anti-c-Met monoclonal antibody), c-Met 1205 (human lgG1 subtype anti-c-Met antibody), and the free toxin SHR152852 were provided by Shanghai Hengrui Pharmaceutical Co., Ltd., a subsidiary company of Jiangsu Hengrui Medicine, Co., Ltd. c-Met protein derived from various species (human, Cynomolgus monkey, and mouse), human FcRn and human Fcy receptors (including FcyR I/CD64, FcyR IIA/CD32a, FcyR IIB/C/CD32b/c, and FcyR III/CD16), and human complement $\mathrm{C} 1 \mathrm{q}$ were purchased from Sino Biological Inc. (Beijing, China), R\&D Systems (Minneapolis, USA), and Abcam (Cambridge, USA), respectively. Biacore ${ }^{\mathrm{TM}}$ Sensor Chip Protein A and Series S Sensor Chip CM5 were supplied by GE Healthcare (Buckinghamshire, UK). Dylight ${ }^{\mathrm{TM}} 488$ NHS Ester and LysoTracker Deep Red were obtained from Thermo Fisher Scientific (Rockford, USA), and Calcein-AM was from Dojindo Molecular Technologies, Inc. (Kumamoto, Japan). Primary antibodies against phosphorylated c-Met (p-c-Met), c-Met, phosphorylated Akt (p-Akt), Akt, phosphorylated ERK ( $p-E R K), E R K$, and $\beta$-tubulin were purchased from Cell Signaling Technology (Danvers, USA), Santa Cruz Biotechnology (Texas, USA), and Sigma (Saint Louis, USA), respectively. Secondary antibodies for Western blots were purchased from Bio-Rad (Hercules, USA).

\section{Cell lines}

The in vitro effects of SHR-A1403 were tested against multiple human cancer cells, including $\mathrm{NCl}-\mathrm{H} 1993, \mathrm{NCl}-\mathrm{H} 441$, and $\mathrm{A} 549$ lung cancer cells, MKN-45 and NCI-N87 gastric cancer cells, Hs578T breast cancer cells, PC-3 prostate cancer cells, Caki-1 renal cancer cells, and HCCLM3 hepatic cancer cells. MKN-45 cells were purchased from the cell bank of the Chinese Academy of Sciences, and all other cell lines were from ATCC (American Type Culture Collection). Cells were cultured in RPMI1640 or DMEM (Hyclone, Logan, USA) supplemented with 10\% FBS (Hyclone) before treatment with test agents.

\section{Biacore assays}

Biacore assays were used to detect the in vitro binding affinity of SHR-A1403 and SHR-A1403 mAb to c-Met protein derived from human, cynomolgus monkey, and mouse. After SHR-A1403 or SHR-A1403 mAb was captured by the Protein A sensor chip, c-Met protein diluted to different concentrations was injected and allowed to flow over the chip surface. The real-time signals of association and dissociation were measured. The data were fitted by a Langmuir model (BIA evaluation version 4.1, $\mathrm{GE}$ ), and the affinity to c-Met protein, as measured by the $K_{\mathrm{D}}$, was obtained.

To measure the binding affinity of SHR-A1403 and SHR-A1403 mAb to human Fcy receptors (FcyRs), FcRn, or C1q, His-tagged FcyRs, FcRn, or C1q proteins were immobilized to the CM5 sensor chip surface, and SHR-A1403 or SHR-A1403 mAb was injected and allowed to flow over the chip surface. The real-time signals of association and dissociation were measured. The data were fitted by a steady-state affinity model (Biacore T200 Evaluation Software version 3.0,GE), and the affinity to $\mathrm{Fc \gamma Rs}, \mathrm{FcRn}$, or $\mathrm{C1q}$ was obtained.

\section{Cell proliferation assay}

Cancer cells were incubated with SHR-A1403, SHR-A1403 mAb, or SHR152852 at different concentrations for $72 \mathrm{~h}$ at $37^{\circ} \mathrm{C}$, and cell viability was measured using the SRB (Sigma, Santa Louis, USA) or CCK-8 (Beyotime Biotechnology, Shanghai, China) method. The $\mathrm{IC}_{50}$ was calculated to evaluate the anti-tumor activity.

Binding affinity with $\mathrm{MKN}-45$ cells

SHR-A1403 and SHR-A1403 mAb at various concentrations were labeled with Dylight ${ }^{\mathrm{TM}} 488 \mathrm{NHS}$ Ester and then incubated with MKN-45 cells on ice for $1 \mathrm{~h}$ with protection from light. After centrifugation, the supernatant was discarded, the cells were resuspended in phosphate buffered saline (PBS), and fluorescence was measured using a FACS Calibur flow cytometer (BD Biosciences, San Jose, USA).

\section{Western blots}

Cells or tumor tissues were collected and lysed in $1 \times$ SDS gel sampling buffer [50 mM Tris- $\mathrm{HCl}(\mathrm{pH} 6.8), 100 \mathrm{mM}$ DTT, 2\% SDS, $10 \%$ glycerol, and $0.1 \%$ bromophenol blue]. The lysate was denatured in boiling water prior to SDS-PAGE electrophoresis. After electrophoresis, the proteins on the gel were transferred onto a PVDF membrane, which was then incubated with primary and secondary antibodies. Signal was detected using ECL reagents (GE Healthcare, Buckinghamshire, UK).

Endocytosis in MKN-45 cells DyLight $^{\text {TM }} 488$ NHS Ester dye-labeled SHR-A1403 or SHR-A1403 $\mathrm{mAb}(10 \mu \mathrm{g} / \mathrm{mL})$ was incubated with MKN-45 cells on ice for $1 \mathrm{~h}$. Subsequently, cells were cultured in fresh medium supplemented with $10 \% \mathrm{FBS}$ at $37^{\circ} \mathrm{C}$ for $0.5,1,2$, or $3 \mathrm{~h}$, transferred immediately to ice to terminate the reaction, centrifuged, re-suspended in stripping buffer, and kept at room temperature for $7 \mathrm{~min}$. The cells were then centrifuged and washed once with FACS buffer before performing FACS analysis.

Lysosomal translocation in MKN-45 cells

MKN-45 cells were seeded in 6-well plates and incubated with Dylight $^{\mathrm{TM}} 488$ NHS Ester dye-labeled SHR-A1403 or SHR-A1403 $\mathrm{mAb}(1 \mu \mathrm{g} / \mathrm{mL})$ at $37{ }^{\circ} \mathrm{C}$ for $23 \mathrm{~h}$. Subsequently, a lysosomemarking red fluorescence probe (LysoTracker Deep Red) was added and incubated with cells at $37^{\circ} \mathrm{C}$ for $1 \mathrm{~h}$. As a control, MKN45 cells were incubated with LysoTracker Deep Red at $37^{\circ} \mathrm{C}$ for $1 \mathrm{~h}$ before adding Dylight ${ }^{\mathrm{TM}} 488$ NHS Ester dye-labeled SHR-A1403 or SHR-A1403 mAb $(1 \mu \mathrm{g} / \mathrm{mL})$ and incubating at $4{ }^{\circ} \mathrm{C}$ for $10 \mathrm{~min}$. After incubation, the supernatant was discarded; cells were washed in PBS three times and observed with a laser scanning confocal microscope.

Cell cycle analysis

MKN-45 cells were seeded in 6-well plates and incubated with SHR-A1403, SHR-A1403 mAb, or SHR152852 for 48 h. Cells were then collected and fixed in ethanol overnight at $-20{ }^{\circ} \mathrm{C}$. Subsequently, RNase was added to remove RNA, and DNA was 
stained with $\mathrm{PI}$ (propidium iodide) for $30 \mathrm{~min}$ at $37^{\circ} \mathrm{C}$ with protection from light. The intracellular DNA content in $1 \times 10^{4}$ cells was quantified using a FACS Calibur flow cytometer, and the data were analyzed using ModFit Lt Mac V3.0 software.

Antibody-dependent cellular cytotoxicity (ADCC) and

complement-dependent cytotoxicity (CDC) assays

In the ADCC assay, Calcein-AM dye-labeled MKN-45 cells were mixed with SHR-A1403, SHR-1403 mAb, or c-Met 1205 and then incubated with human peripheral blood mononuclear cells (PBMCs) (target cells: effector cells ratio was 1:50) for $4 \mathrm{~h}$. After incubation, cells were centrifuged, and the supernatant fluorescence was measured at 490/515 nm. The cell pellets were lysed with $0.1 \%$ Triton $\mathrm{X}-100$ to measure total fluorescence intensity. The cell lysis rate (\%) was calculated using the fluorescence intensity and evaluated as the ADCC activity of the test articles.

In the CDC assay, serum samples were first separated from whole blood samples collected from two healthy volunteers (donor $\mathrm{A}$ and $\mathrm{B}$ ); half of each sample was incubated in a $56^{\circ} \mathrm{C}$ water bath for 30 min to inactivate the complement, and the other half was kept on ice until use. MKN-45 cells were seeded in 96-well plates at $1 \times 10^{5}$ cells per well with RPMI1640 culture medium supplemented with $10 \%$ low-lgG FBS and then treated with SHRA1403, SHR-A1403 mAb, or c-Met 1205 for 15 min. Subsequently, cells were incubated with the prepared human serum samples (final concentration of $12.5 \%$ ) for $2 \mathrm{~h}$. After incubation, the cells were lysed with $0.1 \%$ Triton X-100 lysis buffer for $15 \mathrm{~min}$, and CellTiter-Glo ${ }^{\oplus}$ Reagent (Promega, Madison, USA) was added to measure $O D$ values on the microplate reader and calculate the lysis rate (\%).

In vivo xenograft anti-tumor activity

Male BALB/CA-nude mice (6-7 weeks old) were used to evaluate the in vivo anti-tumor efficacy of the test agents. Animals were acclimated for at least one week before each experiment and kept in the animal room with 12-h light: 12-hour dark cycles. All experiments were performed in compliance with the Institutional Animal Care and Use Committee (IACUC) at Shanghai Institute of Materia Medica, Chinese Academy of Sciences, following the guidance of the Association for Assessment and Accreditation of Laboratory Animal Care (AAALAC). HCCLM3, NCl-H1993, or MKN45 cells were implanted subcutaneously into the right flank region of each mouse. Each group consisted of 10 tumor-bearing mice. Once tumors reached an average volume of $125 \mathrm{~mm}^{3}$, mice were randomized, and test agents were administered via one or more intravenous injections. Mice were observed for signs of morbidity/ mortality throughout the treatment period. Tumor volume was measured two or three times weekly using a caliper. Body weight was measured as a parameter of potential toxicity.

Two MKN-45 xenograft mice studies were performed; one was used to evaluate anti-tumor efficacy after multiple dosing, and the other was used to evaluate the pharmacokinetics (PK)/pharmacodynamics (PD) correlation after a single intravenous dosing. In the PK/PD study, blood samples were collected at different time points to obtain serum for the detection of ADC concentrations using a validated ELISA method and PK parameter calculation. Moreover, tumor tissues were dissected and subjected to Western blot assays to assess c-Met and ERK protein levels.

In vivo anti-tumor activity in HCC patient-derived xenograft (PDX)bearing mice

Samples from the HCC PDX model (WuXi AppTec (Shanghai) Co., Ltd.), originally established from a surgically resected clinical sample, were implanted in BALB/c nude mice and defined as passage 0 (P0). P5 tumor tissue was used for this study. Animals with body weights of 18-22 g were acclimated for at least one week before each experiment and kept in the animal room with 12-hour light: 12-hour dark cycles. All experiments were
Table 1. In vitro binding affinity to c-Met protein derived from different species

\begin{tabular}{lll}
\hline Analyte & c-Met & $K_{\mathrm{D}}(\mathrm{M})$ \\
\hline SHR-A1403 & Human & $1.71 \times 10^{-8}$ \\
SHR-A1403 mAb & & $1.58 \times 10^{-8}$ \\
SHR-A1403 & Cynomolgus monkey & $3.19 \times 10^{-8}$ \\
SHR-A1403 mAb & & $2.84 \times 10^{-8}$ \\
SHR-A1403 & Mouse & No binding \\
SHR-A1403 mAb & & No binding \\
\hline
\end{tabular}

performed in compliance with the IACUC at WuXi AppTec following the guidance of AAALAC. P5 tumor slices $\left(\sim 30 \mathrm{~mm}^{3}\right)$ were implanted subcutaneously in the right flank of each mouse for tumor development. Treatments were started on day 15 after tumor inoculation, at which time the average tumor size was approximately $150 \mathrm{~mm}^{3}$. Each group consisted of 6 tumor-bearing mice, which received twice-weekly intravenous injections of SHRA1403 at 1, 3, or $10 \mathrm{mg} / \mathrm{kg}$ or SHR-A1403 mAb at $10 \mathrm{mg} / \mathrm{kg}$ for two consecutive weeks. Mice were observed for signs of morbidity/ mortality throughout the treatment period. Tumor volume was measured twice weekly until 45 days after treatment. Body weight was also monitored as an index of potential toxicity. At the end of the study, tumor tissues were dissected, c-Met protein expression was measured by immunohistochemistry (IHC), and samples were scored.

\section{RESULTS}

SHR-A1403 showed high binding affinity with c-Met in vitro The binding affinity of SHR-A1403 to c-Met protein derived from human, cynomolgus monkey, and mouse was evaluated by the $K_{\mathrm{D}}$ determined using Biacore assays and compared to that of naked SHR-A1403 mAb. As shown in Table 1, SHR-A1403 possessed high affinity to human and monkey c-Met with $K_{\mathrm{D}} \mathrm{s}$ in the double-digit nanomolar range, which is comparable to that of SHR-A1403 mAb. This result indicated that antibody conjugation with toxin did not appreciably change its binding affinity to c-Met. Neither SHRA1403 nor SHR-A1403 mAb could bind mouse c-Met.

SHR-A1403 potently inhibited the proliferation of human cancer cells expressing different levels of c-Met

The effects of SHR-A1403 on the proliferation of various types of human cancer cells were evaluated and compared with the effects of SHR-A1403 mAb and the free toxin SHR152852. The $\mathrm{IC}_{50}$ values showed that SHR-A1403 had high potency in MKN-45, HCCLM3, and $\mathrm{NCl}-\mathrm{H} 1993$ cells and moderate potency in $\mathrm{NCl}-\mathrm{H} 441, \mathrm{Hs} 578 \mathrm{~T}$, PC-3, and Caki- 1 cells (Table 2). SHR-A1403 weakly inhibited the proliferation of $\mathrm{A} 549$ and $\mathrm{NCl}-\mathrm{N} 87$ cells; the $\mathrm{IC}_{50}$ for these cells was greater than $1 \mu \mathrm{g} / \mathrm{mL}$. In contrast, SHR-A1403 mAb minimally affected the proliferation of other cell lines, as indicated by $\mathrm{IC}_{50}$ values higher than $5 \mu \mathrm{g} / \mathrm{mL}$. However, the free toxin showed significant inhibition of all tested cancer cells; $I C_{50}$ values ranged from 3 to $120 \mathrm{nM}$. These results revealed that after conjugation, SHR-A1403 had higher potency than SHR-A1403 mAb.

c-Met protein expression was quantified in most of the studied cell lines using flow cytometry. The results showed that NCI-N87 cells had relatively low expression; $\mathrm{NCl}-\mathrm{H} 441, \mathrm{Hs} 578 \mathrm{~T}$, and Caki-1 cells had moderate expression; and HCCLM3, NCl-H1993, and MKN-45 cells had relatively high expression (Table 2 ). The protein expression levels of c-Met in these cell lines generally correlated with the anti-tumor potency of SHR-A1403, supporting the concept of target expression-dependent anti-tumor activity of ADCs compared with on-target small molecule inhibitors. 


\begin{tabular}{|c|c|c|c|c|}
\hline \multirow[t]{2}{*}{ Cell Line } & \multirow{2}{*}{$\begin{array}{l}\text { c-Met } \\
\text { expression }^{\mathrm{a}}\end{array}$} & \multicolumn{3}{|c|}{$I C_{50}($ Mean $\pm S D)$} \\
\hline & & $\begin{array}{l}\text { SHR-A1403 } \\
\text { (ng/mL) }\end{array}$ & $\begin{array}{l}\text { SHR-A1403 } \\
\mathrm{mAb} \\
(\mathrm{ng} / \mathrm{mL})\end{array}$ & $\begin{array}{l}\text { SHR152852 } \\
\text { (nM) }\end{array}$ \\
\hline \multicolumn{5}{|l|}{ Lung cancer } \\
\hline $\mathrm{NCl}-\mathrm{H} 1993$ & 287 & $16.3 \pm 4.1$ & $6200 \pm 1300$ & $28.9 \pm 1.3$ \\
\hline $\mathrm{NCl}-\mathrm{H} 441$ & 80.6 & $46.7 \pm 39.4$ & $>10000$ & $77.6 \pm 12.1$ \\
\hline A549 & $N D^{b}$ & $1987.5 \pm 164.8$ & $>10000$ & $27.1 \pm 1.4$ \\
\hline \multicolumn{5}{|c|}{ Gastric cancer } \\
\hline MKN-45 & 227 & $7.8 \pm 3.1$ & $>10000$ & $10.4 \pm 5.1$ \\
\hline NCl-N87 & 14.4 & $>10000$ & $>10000$ & $36.2 \pm 1.4$ \\
\hline \multicolumn{5}{|c|}{ Breast cancer } \\
\hline $\mathrm{Hs578T}$ & 40.3 & $53.9 \pm 15.2$ & $>10000$ & $94.4 \pm 3.8$ \\
\hline \multicolumn{5}{|c|}{ Prostate cancer } \\
\hline PC-3 & ND & $78.2 \pm 19.5$ & $>10000$ & $89.5 \pm 15.2$ \\
\hline \multicolumn{5}{|c|}{ Renal cancer } \\
\hline Caki-1 & 36.3 & $214.8 \pm 84.4$ & $>10000$ & $117.7 \pm 2.1$ \\
\hline \multicolumn{5}{|c|}{ Hepatic cancer } \\
\hline HCCLM3 & 438 & $3.2 \pm 0.0$ & $>10000$ & $3.1 \pm 0.4$ \\
\hline
\end{tabular}

SHR-A1403 exhibited favorable PK/PD correlation in MKN-45 cell xenograft mice

In this study, MKN-45 xenograft-bearing mice were given a single dose of SHR-A1403 at 1 or $3 \mathrm{mg} / \mathrm{kg}$ via intravenous injection, and the intratumoral c-Met protein levels and serum SHR-A1403 levels were assessed to establish the PK and PD relationship. Figure 1 illustrates that a single injection of SHR-A1403 time-dependently decreased c-Met protein levels in tumors, and this downregulation was maintained until $168 \mathrm{~h}$ after treatment; the maximal effects were achieved earlier at higher dose. The exposure to SHR-A1403 was proportional to the dose administered. The $C_{\max }$ and concentration measured $168 \mathrm{~h}$ after injection indicated slow metabolism/clearance of SHR-A1403 after administration (Table 3), and these values exhibited a favorable correlation with changes of intratumoral c-Met expression.

SHR-A1403 showed potent anti-tumor activity in xenograft mouse models

The anti-tumor efficacy of SHR-A1403 was further evaluated in xenograft mice bearing cancer cells with high c-Met expression, including hepatic cancer HCCLM3, lung cancer $\mathrm{NCl}-\mathrm{H} 1993$, and gastric cancer MKN-45 cells, and the effects were compared with the effects of SHR-A1403 mAb, the free toxin, or their combination. In the HCCLM3 xenograft model, a single administration of SHR-A1403 could dose-dependently delay tumor growth; terminal tumor growth inhibition (TGl) of $36 \%, 87 \%$, and $178 \%$ was seen at doses of 1,3 , and $10 \mathrm{mg} / \mathrm{kg}$, respectively. At $10 \mathrm{mg} / \mathrm{kg}, 30 \%$ PR (partial response) and 60\% CR (complete response) were achieved, and tumor regression could be sustained until 42 days after treatment. In contrast, treatment with $10 \mathrm{mg} / \mathrm{kg}$ SHR-A1403 mAb only had minor efficacy in this model (Fig. 2a and Table 4). In the $\mathrm{NCl}-\mathrm{H} 1993$ xenograft model, a single injection of SHR-A1403 dosedependently delayed tumor growth; terminal TGI of $45 \%, 63 \%$, and $124 \%$ was observed at doses of 1,3 , and $10 \mathrm{mg} / \mathrm{kg}$, respectively. At $10 \mathrm{mg} / \mathrm{kg}, 70 \% \mathrm{PR}$ was achieved, and tumor regression could be sustained until 34 days after treatment. In comparison, SHR-A1403 mAb only yielded minor efficacy with TGI of $42 \%$ (Fig. $2 \mathrm{~b}$ and Table 4). In the MKN-45 xenograft model, a
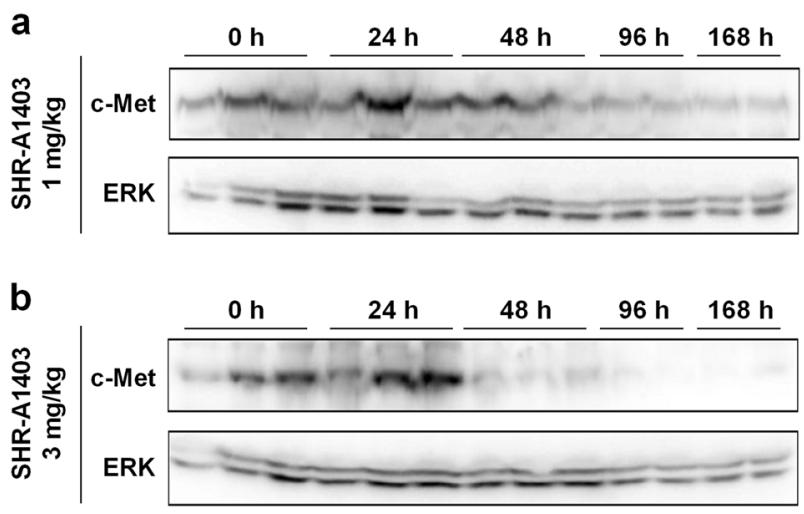

Fig. 1 Inhibition of intratumoral c-Met expression by SHR-A1403 in an MKN-45 cell xenograft model. Mice bearing MKN-45 cells were administered with a single intravenous injection of SHR-A1403 at 1 or $3 \mathrm{mg} / \mathrm{kg}$, and tumor samples were collected prior to injection and $24,48,96$, and $168 \mathrm{~h}$ after injection. c-Met protein levels in tumor tissues were detected by Western blotting. Representative bands are shown. a Mice were treated with SHR-A1403 at $1 \mathrm{mg} / \mathrm{kg}$. b Mice were treated with SHR-A1403 at $3 \mathrm{mg} / \mathrm{kg}$

Table 3. Serum exposure of SHR-A1403 in MKN-45 xenograft mice model following single intravenous injection

\begin{tabular}{llll}
\hline Dose & $\begin{array}{l}C_{\max } \\
(\mu \mathrm{g} / \mathrm{mL})\end{array}$ & $\begin{array}{l}\mathrm{AUC}_{0-168 \mathrm{~h}} \\
(\mu \mathrm{g} / \mathrm{mL} * \mathrm{~h})\end{array}$ & $\begin{array}{l}\text { Serum conc. at } 168 \mathrm{~h} \text { postdose } \\
(\text { Mean } \pm \mathrm{SD} ; \mu \mathrm{g} / \mathrm{mL})\end{array}$ \\
\hline $1 \mathrm{mg} / \mathrm{kg}$ & 14.08 & 937.72 & $3.1 \pm 1.3$ \\
$3 \mathrm{mg} / \mathrm{kg}$ & 49.93 & 2663.07 & $10.2 \pm 1.2$ \\
\hline
\end{tabular}

$C_{\max }$, peak concentration in serum, $A U C$ area under the concentration-time curve, conc. concentration

single administration of SHR-A1403 dose-dependently delayed tumor growth; terminal TGI of $51 \%, 99 \%$, and $149 \%$ was observed at doses of 1,3 , and $10 \mathrm{mg} / \mathrm{kg}$, respectively. At 3 and $10 \mathrm{mg} / \mathrm{kg}$, $60 \%$ PR and $100 \%$ PR were achieved, respectively, and tumor regression could be sustained until 21 days after treatment. However, SHR-A1403 mAb and the free toxin SHR152852 only yielded minor efficacy with TGI of 20 and 56\%, respectively. Unsurprisingly, no synergistic inhibition of tumor growth was observed upon the combined injection of SHR-A1403 mAb and the free toxin SHR152852 (Fig. 2C and Table 4). No significant impact on body weight was seen in any model with any of these treatments throughout the study period, suggesting that all the treatments are well tolerated. These results revealed that SHR-A1403 had superior anti-tumor potency without increased toxicity compared with c-Met antibody, the free toxin, or their combination.

SHR-A1403 yielded significant anti-tumor activity in an HCC PDX model with confirmed c-Met overexpression

In this model, tumor-bearing mice were given vehicle, SHR-A1403 $(1,3$, or $10 \mathrm{mg} / \mathrm{kg})$, or SHR-A1403 mAb $(10 \mathrm{mg} / \mathrm{kg})$ via twiceweekly intravenous injection for two consecutive weeks, and the study was conducted for 45 days after treatment. Over the whole period, no body weight loss was noted for any group, indicating no obvious toxicity caused by the treatments. As illustrated in the tumor growth curves (Fig. 3a) and the TGI detailed in Table 5, SHRA1403 mAb minimally delayed tumor growth, whereas SHR-A1403 at all dose levels strongly inhibited tumor growth (regression). CR was nearly achieved after four injections at doses of 3 or $10 \mathrm{mg} / \mathrm{kg}$, 
a

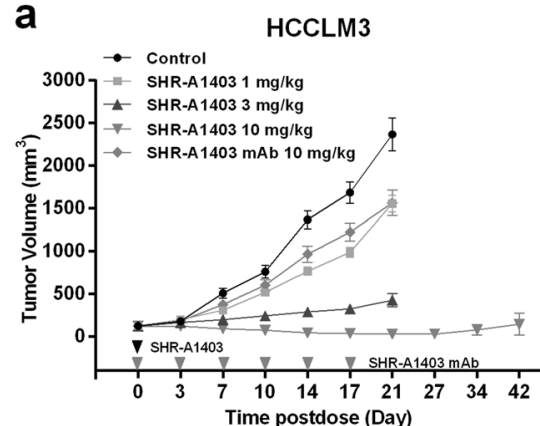

b

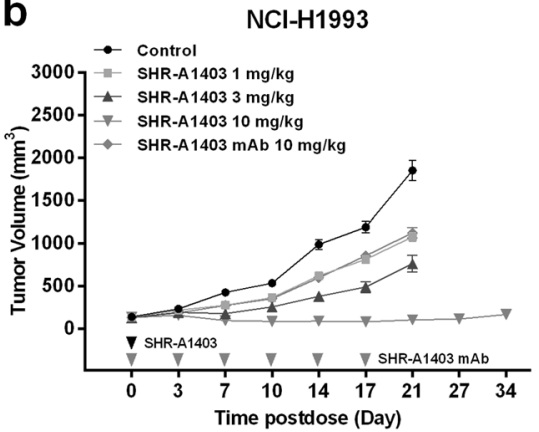

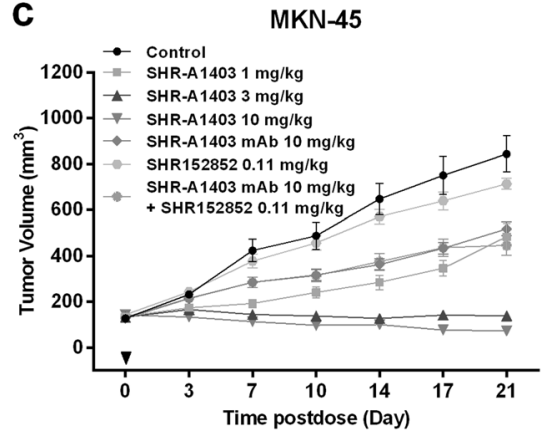

Fig. 2 In vivo anti-tumor activity of SHR-A1403 in mouse xenograft models. a-c BALB/cA-nude mice were subcutaneously transplanted with HCCLM3 (a), NCl-H1993 (b), or MKN-45 (c) cells. Once tumors reached an average volume of $125 \mathrm{~mm}^{3}$, mice were randomized $(n=10$ per group) and given test agents at indicated doses via intravenous injection. The dosing frequency for indicated treatment is shown by triangular arrowheads in the figures. Tumor size was measured twice weekly and plotted against observation day for each group. The data are shown as the means \pm SEM

\begin{tabular}{|c|c|c|c|c|}
\hline \multirow[t]{4}{*}{ HCCLM3 } & Vehicle & IV/D0 & - & \\
\hline & SHR-A1403/1 mg/kg & IV/DO & 36 & \\
\hline & SHR-A1403/3 mg/kg & IV/D0 & 87 & 1/10 PR \\
\hline & SHR-A1403/10 mg/kg & IV/D0 & 178 & $3 / 10 \mathrm{PR} ; 6 / 10 \mathrm{CR}$ \\
\hline \multirow{4}{*}{$\mathrm{NCl}-\mathrm{H} 1993$} & SHR-A1403/1 mg/kg & IV/DO & 45 & \\
\hline & SHR-A1403/3 mg/kg & IV/D0 & 63 & \\
\hline & SHR-A1403/10 mg/kg & IV/D0 & 124 & 7/10 PR \\
\hline & SHR-A1403 mAb/10 mg/kg & IV/D0, 3, 7, 10, 14, 17 & 42 & \\
\hline \multirow[t]{4}{*}{ MKN-45 } & Vehicle & IV/D0 & - & \\
\hline & SHR-A1403 mAb/10 mg/kg & IV/D0 & 45 & \\
\hline & SHR152852/0.11 mg/kg & IV/D0 & 20 & \\
\hline & $\begin{array}{l}\text { SHR-A1403 mAb/10 mg } / \mathrm{kg} \\
+ \\
\text { SHR152852/0.11 mg/kg }\end{array}$ & IV/D0 & 56 & \\
\hline
\end{tabular}

and the effect was maintained until the end of the study; the 3 $\mathrm{mg} / \mathrm{kg}$ treatment achieved maximum efficacy without needing dose escalation.

To further confirm the mechanisms through which SHR-A1403 arrested in vivo tumor growth, tumor samples were collected, and a c-Met expression score was obtained. As the IHC staining images in Fig. 3b show, the PDX model used in this study highly expressed c-Met and was scored as $3+$, demonstrating its suitability for our study and good correlation with the observed remarkable anti-tumor activity of SHR-A1403. The study revealed potent anti-tumor activity of SHR-A1403 in the PDX model with high intratumoral c-Met expression, which offers a superior reference for clinical trials.

Cellular mechanisms mediating the anti-tumor potency of SHRA1403

The mechanisms of SHR-A1403 anti-tumor activity were explored from the aspects of cell surface c-Met binding, downstream signaling transduction, endocytosis, intracellular translocation, the induction of microtubule depolymerization, cell cycle arrest, and ADCC and CDC activities.

MKN-45 cells with high c-Met expression were employed for binding assays using a flow cytometry method. SHR-A1403 bound to MKN-45 cells in a dose-dependent manner comparable to that of SHR-A1403 mAb (Fig. 4a). Functional blockage of c-Met by SHRA1403 or SHR-A1403 mAb dose-dependently down-regulated the levels of phosphorylated c-Met, Akt, and ERK, with equivalent potency at the same concentrations (Fig. 4b). Because SHR-A1403 $\mathrm{mAb}$ minimally affected cancer cell proliferation but showed comparable inhibition of the c-Met pathway, this result demonstrated that blockage of the c-Met signaling pathway upon binding with c-Met was not the predominant contributor to the high anti-tumor efficacy of SHR-A1403. The decreased c-Met levels (Fig. 4b) after treatment with SHR-A1403 or SHR-A1403 mAb provided clues regarding the endocytosis of c-Met. Dylight ${ }^{\mathrm{TM}} 488$ NHS Ester-labeled SHR-A1403 or SHR-A1403 mAb was used to 


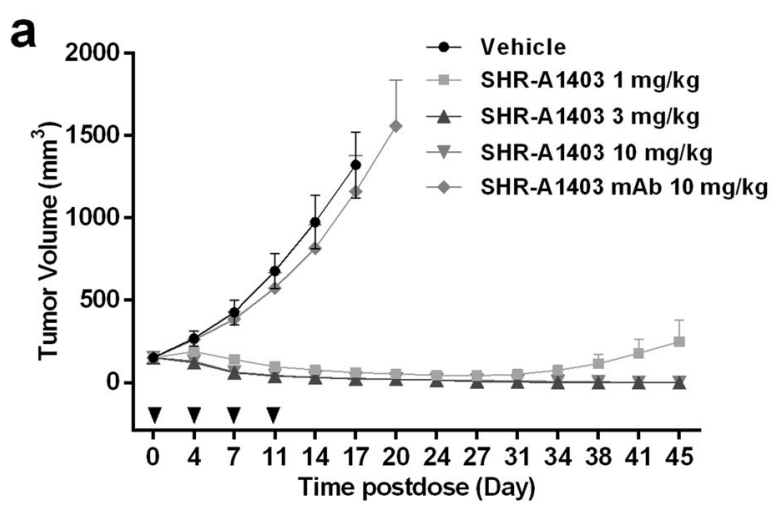

b

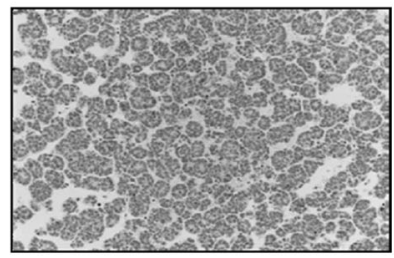

Normal liver tissue $20 \times$, score $=0$

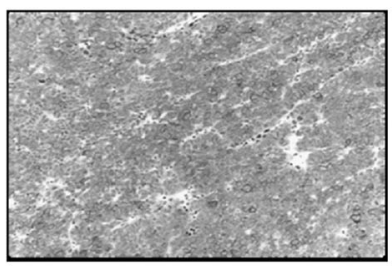

HCC tissue
$20 \times$, score $=3+$

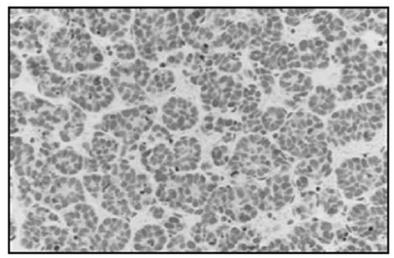

Normal liver tissue $40 \times$, score $=0$

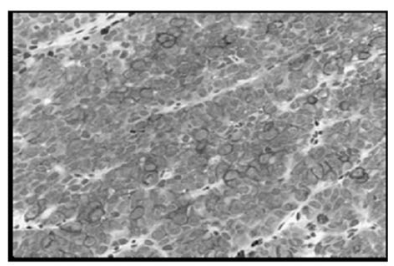

HCC tissue

$40 \times$, score $=3+$
Fig. 3 In vivo anti-tumor activity of SHR-A1403 in PDX model. HCC tumor cells derived from patients (passage 5) were implanted subcutaneously in BALB/C nude mice at an initial tumor size of approximately $30 \mathrm{~mm}^{3}$. On day 15 after transplantation, animals were randomized ( $n=6$ per group) and treated with test agents at indicated doses via intravenous injection. The dosing frequency is shown by triangular arrowheads in the figures. a Tumor size was measured twice weekly and plotted against observation day for each group. The data are shown as the means \pm SEM. b Representative images of IHC staining for c-Met in liver tissues. Upper panel, liver tissues from normal mice as negative control; lower panel, tumor tissues collected from mice used in this study. The score was evaluated according to c-Met staining and was proportional to intratumoral c-Met protein expression. Left panel, images captured at 20x magnification; right panel, images captured at $40 \times$ magnification

track internalization (endocytosis) and cellular translocation after binding c-Met. The results showed that SHR-A1403 could be timedependently internalized into MKN-45 cells with a maximal internalization rate of approximately $40 \%$, and SHR-A1403 mAb exhibited similar endocytosis with a slightly lower internalization rate (Fig. 4c). After incubation for $10 \mathrm{~min}$, the labeled SHR-A1403 or SHR-A1403 mAb was predominantly located on the cell membrane (Fig. 4d, first and third rows) and was then transported to the lysosome as exhibited by the co-localization of Dylight ${ }^{\mathrm{TM}}$ 488 and the LysoTracker $24 \mathrm{~h}$ after treatment (Fig. $4 \mathrm{~d}$, second and forth rows).

In the lysosome, toxin-related metabolites of SHR-A1403 were identified in MKN-45 cells, and these levels increased as incubation time increased, suggesting the hydrolysis of SHR-A1403 by lysosomal enzymes. To determine whether the observed anti-tumor efficacy

\begin{tabular}{|c|c|c|}
\hline \multirow[t]{2}{*}{ Treatment } & \multicolumn{2}{|c|}{ Tumor size $\left(\mathrm{mm}^{3}\right)$} \\
\hline & Day 17 & Day 45 \\
\hline Vehicle & $1320 \pm 201$ & $\rho^{\mathrm{a}}$ \\
\hline SHR-A1403-1 mg/kg & $60 \pm 7$ & $53 \pm 6$ \\
\hline SHR-A1403-3 mg/kg & $22 \pm 4$ & $20 \pm 4$ \\
\hline SHR-A1403-10 mg/kg & $24 \pm 9$ & $20 \pm 8$ \\
\hline SHR-A1403 mAb-10 mg/kg & $1159 \pm 221$ & $\rho^{\mathrm{a}}$ \\
\hline
\end{tabular}

${ }^{a}$ Mice were killed on Day 45 due to heavy tumor burden. The data are shown as mean \pm SEM. Animal number, $n=6$ per group

$H C C$ hepatocellular carcinoma, $P D X$ patient-derived xenograft

was mediated by toxin-related substances, we studied the effects of SHR-A1403 on microtubule aggregation and cell cycle distribution and compared the effects from free toxin and SHR-A1403 mAb in MKN-45 cells. Figure 4 e shows that both SHR-A1403 and free toxin similarly dose-dependently decreased the levels of polymeric $\beta$-tubulin. Similarly, both SHR-A1403 and free toxin dosedependently and remarkably induced a cell cycle arrest, as indicated by more cells at the G2/M phase after incubation (Fig. 4f). Additionally, five toxin-related metabolites identified in MKN-45 cells also potently inhibited microtubule aggregation with $\mathrm{IC}_{50} \mathrm{~S}$ of 2.6, 3.0, 0.3, 0.3, and $0.4 \mu \mathrm{M}$, respectively. In contrast, SHR-A1403 $\mathrm{mAb}$ had no effects on microtubular aggregation or cell cycle arrest.

The binding of SHR-A1403 with Fc receptors and C1q and the resultant $A D C C$ and $C D C$ activities were measured to further clarify the involved mechanisms. In vitro binding affinity revealed that both SHR-A1403 and SHR-A1403 mAb had low affinity for FcRn, FcyRs (FcyR I, FcyR IIA, FcyR IIB/C, and FcyR III) or C1q (Table 6). Correspondingly, SHR-A1403 and SHR-A1403 mAb showed approximately no ADCC activity (maximal lysis rate was 2.6 and $0 \%$, respectively), whereas the positive control c-Met 1205 , a human IgG1 anti-c-Met antibody, showed a maximal ADCC activity (lysis rate) of $27.8 \%$. The CDC activity of SHR-A1403 (12.04\% for Donor A and 14.19\% for Donor B) and SHR-A1403 mAb (15.15\% for Donor $A$ and $16.83 \%$ for Donor B) was also lower than that of CMet 1205 (25.31\% for Donor A and 27.44\% for Donor B). These results demonstrated that $A D C C$ and $C D C$ were scarcely involved in the anti-tumor activity of SHR-A1403 or SHR-A1403 mAb, which is in accord with their low affinity for Fc receptors and $\mathrm{C} 1 \mathrm{q}$ and the nature of the lgG2 subtype of the c-Met antibody as designed.

These collective data further verified our speculation that the toxin-related mechanisms, i.e., the inhibition of microtubule aggregation and subsequent cell cycle arrest, mediated the antitumor effects of SHR-A1403 instead of the isolated inhibition of the c-Met signaling pathway caused by the naked antibody.

\section{DISCUSSION}

Therapies targeting c-Met, either small molecules or antibody inhibitors, have long been investigated for treating solid tumors $[1,6,7]$. However, limited benefits were achieved in clinical trials. For these therapies, emerging evidence reveals that gene amplification and/or constitutively activated downstream signaling are more critical indicators of efficacy than c-Met protein overexpression $[16,17]$. This understanding is supported by the fact that the clinical benefit of c-Met antibody ABT-700 was only noted in patients with c-Met gene amplification[21]. A lowfrequency of gene amplification in solid tumors and difficulties in selecting screening biomarkers of downstream signaling raise challenges for the development of novel, effective small molecule or antibody inhibitors. The ADC strategy could theoretically overcome these limitations because c-Met protein overexpression 
a

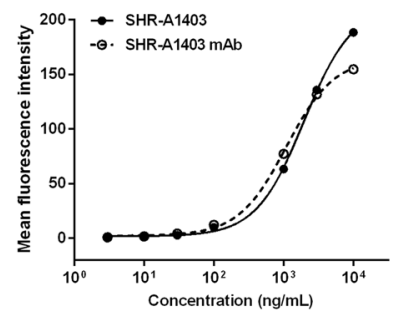

d

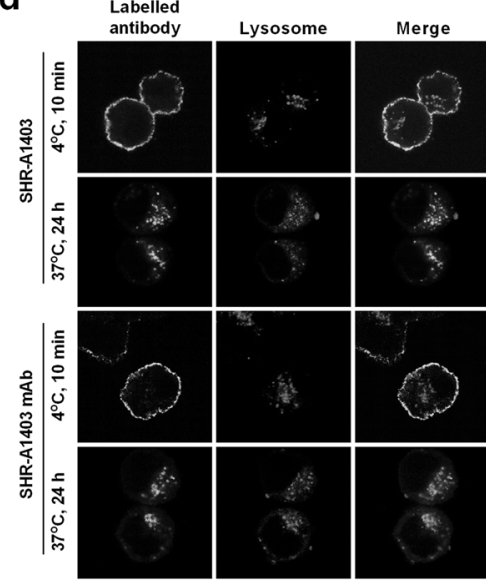

e

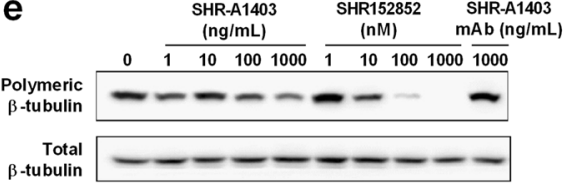

b

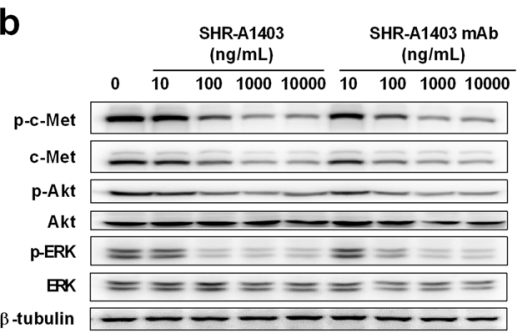

C

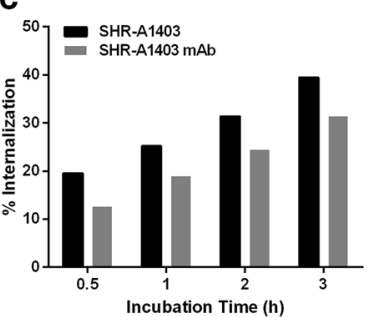

f

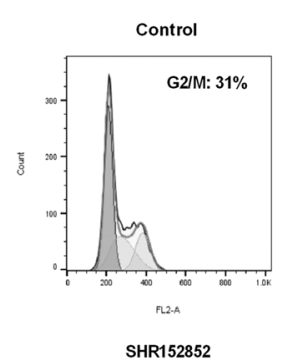

$0.01 \mu \mathrm{M}$

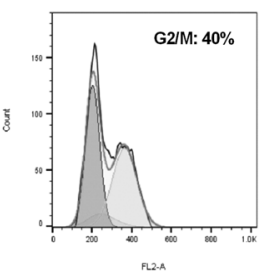

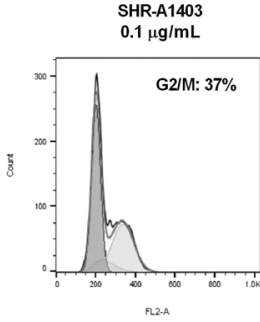

SHR152852

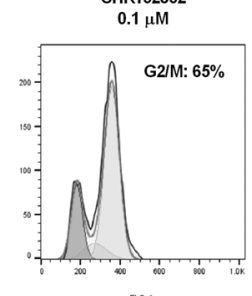

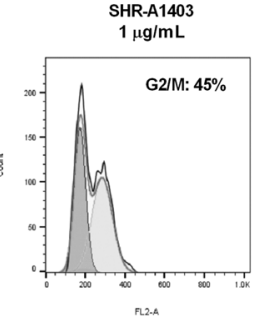
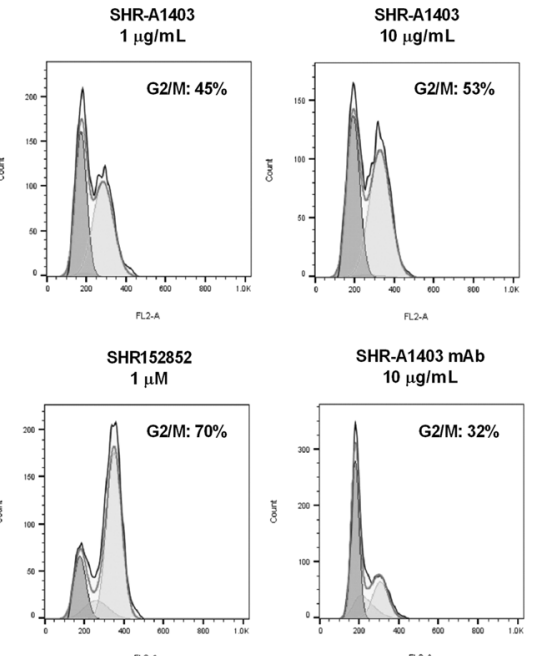

Fig. 4 Cellular mechanisms underlying the anti-tumor activity of SHR-A1403. a Binding affinity of SHR-A1403 and SHR-A1403 mAb to MKN-45 cells measured by flow cytometry at different concentrations. Statistical analysis was conducted using Student's $t$ test. b Inhibitory effects of SHR-A1403 and SHR-A1403 mAb on c-Met downstream signaling. Levels of phosphorylated and total c-Met, Akt, and ERK protein in MKN-45 cells were detected by Western blotting after incubation with SHR-A1403 or SHR-A1403 mAb at different concentrations for $24 \mathrm{~h}$. Representative bands are shown. c Endocytosis rate of SHR-A1403 and SHR-A1403 mAb in MKN-45 cells. After incubation with Dylight ${ }^{\mathrm{TM}} 488-$ labeled SHR-A1403 or SHR-A1403 mAb for $0.5,1,2$, or $3 \mathrm{~h}$, intracellular fluorescence was detected by flow cytometry. d Translocation of SHRA1403 or SHR-A1403 mAb to lysosomes in MKN-45 cells. For short-term incubation, cells were first incubated with LysoTracker Deep Red at $37^{\circ} \mathrm{C}$ for $1 \mathrm{~h}$ followed by treatment with Dylight ${ }^{\mathrm{TM}} 488 \mathrm{NHS}$ Ester-labeled SHR-A1403 or SHR-A $1403 \mathrm{mAb}$ at $4{ }^{\circ} \mathrm{C}$ for $10 \mathrm{~min}$. For long-term incubation, cells were first treated with Dylight ${ }^{\mathrm{TM}} 488 \mathrm{NHS}$ Ester-labeled SHR-A1403 or SHR-A1403 mAb at $37{ }^{\circ} \mathrm{C}$ for $23 \mathrm{~h}$ followed by incubation with LysoTracker Deep Red at $37^{\circ} \mathrm{C}$ for an additional $1 \mathrm{~h}$. After treatment, cell slides were prepared and observed under a confocal microscope. Representative images are presented here. e Effects of SHR-A1403, SHR-A1403 mAb, and SHR152852 on microtubule aggregation. MKN-45 cells were incubated with SHR-A1403, SHR-A1403 mAb, or SHR152852 for $48 \mathrm{~h}$, and the intracellular levels of polymeric and total $\beta$ tubulin were determined by Western blotting. Representative bands are shown. f Effects of SHR-A1403, SHR-A1403 mAb, or SHR152852 on MKN-45 cell cycle distribution. MKN-45 cells were incubated with SHR-A1403, SHR-A1403 mAb, or SHR152852 for 48 h, and cell cycle distribution was analyzed by flow cytometry. The percentage of cells in G2/M phase was calculated as an indicator of cell cycle arrest

is sufficient to mediate the anti-tumor efficacy of the potent toxic small molecules by targeting the c-Met-overexpressing tumors. ABBV-399 from Abbvie, the only c-Met ADC in clinical trials, has shown acceptable tolerance and preliminary clinical benefit in a Phase 1 trial, suggesting that c-Met ADC is a novel, promising anticancer therapy. Here, we present the preclinical in vitro and in vivo efficacy of a novel c-Met ADC, SHR-A1403.

SHR-A1403 is composed of a c-Met-targeting monoclonal antibody (SHR-A1403 mAb) conjugated to a novel cytotoxin (SHR152852), which is distinguished from MMAE. After conjugation, the binding affinity of the antibody to c-Met was not changed, as confirmed by Biacore assay (Table 1). In multiple cancer cell lines, SHR-A1403 showed significant inhibitory effects on cell proliferation, and the potency was generally proportional to c-Met expression, which validates the "targeting" property of ADCs (Table 2). Similar findings were also reported with ABBV-399 in both preclinical and clinical scenarios $[19,20]$. The results of a
Phase 1 expansion study of ABBV-399 revealed that PR was achieved in $3 / 10$ NSCLC patients with c-Met + (score $\geq 2+$ by IHC), whereas there were no responses among 9 patients with c-Met negative tumors [19]. The anti-tumor activity of SHR-A1403 was further confirmed in three xenograft mouse models in which SHRA1403 elicited prominent efficacy compared with the naked antibody, the free toxin, or their combination (Fig. 2 and Table 4). These results indicated that SHR-A1403 could be a promising therapeutic agent with more benefit in patients who are refractory or unresponsive to the currently available therapies.

In addition to the prominent anti-tumor efficacy, the potential on-target toxicity caused by c-Met protein expressed in normal tissues (e.g., epithelial and endothelial cells, neurons, hepatocytes, and hematopoietic cells) should also be considered [22]. Unlike tumor cells, limited cell killing activity was observed for ABBV-399 in normal cells even with high c-Met protein expression [20]. Moreover, ABBV-399 was reported to exhibit non-adverse or 
Table 6. In vitro binding affinity to human Fc receptors and C1q

\begin{tabular}{|c|c|c|}
\hline Analyte & Fc receptor/C1q & $K_{\mathrm{D}}(\mathrm{M})$ \\
\hline SHR-A1403 & FcRn & $9.43 \times 10^{-6}$ \\
\hline SHR-A1403 mAb & & $1.20 \times 10^{-5}$ \\
\hline SHR-A1403 & $\mathrm{Fc} \gamma \mathrm{R} \mathrm{I} / \mathrm{CD} 64$ & $1.72 \times 10^{-5}$ \\
\hline SHR-A1403 mAb & & $1.79 \times 10^{-5}$ \\
\hline SHR-A1403 & FcyR IIA/CD32a & $>1 \times 10^{-4}$ \\
\hline SHR-A1403 mAb & & $>1 \times 10^{-4}$ \\
\hline SHR-A1403 & $\mathrm{Fc \gamma R}$ IIB/C/CD32b/c & $>1 \times 10^{-4}$ \\
\hline SHR-A1403 mAb & & $>1 \times 10^{-4}$ \\
\hline SHR-A1403 & FcyR III/CD16 & $5.86 \times 10^{-5}$ \\
\hline SHR-A1403 mAb & & $5.78 \times 10^{-5}$ \\
\hline SHR-A1403 & $\mathrm{C} 1 \mathrm{q}$ & $>1 \times 10^{-4}$ \\
\hline SHR-A1403 mAb & & $>1 \times 10^{-4}$ \\
\hline
\end{tabular}

reversible toxicities in healthy cynomolgus monkeys receiving repeated dosing [20]. A similar safety profile was observed for SHR-A1403 from extensive preclinical toxicity studies in healthy rats and monkeys. In addition, the cell permeability of the free toxin SHR152852 was relatively low, and therefore, limited free toxins/toxin-related metabolites would transport back to the circulation after being hydrolyzed and released in the targeted cells. Collectively, these findings suggest an acceptable safety profile of c-Met ADC and provide references for risk control and the study design of trials in humans.

The anti-tumor efficacy of ADCs primarily relies on the conjugated toxin instead of inhibition of target-related signaling [23-25]. Here, the detailed underlying mechanisms were elucidated in in vitro studies (Fig. 4). Upon binding with c-Met on the cell membrane, the downstream c-Met signaling pathway was blunted as shown by decreased protein levels of critical downstream effectors, i.e., phosphorylated Akt and ERK, and simultaneously, endocytosis and translocation to lysosomes mediated by an antibody-c-Met complex were triggered. The endocytosis and translocation processes for ADCs have been studied and explained previously [23-25]. In the lysosomes, the conjugated toxin is dissociated from the ADC by intra-lysosomal enzymes, as supported by the detection of the free toxin and related metabolites in the cells. As cytotoxic microtubule inhibitors, the free toxin and related metabolites significantly inhibited microtubule aggregation and subsequently induced a cell cycle arrest. However, limited ADCC and CDC activities were observed for SHRA1403 or SHR-A1403 mAb, and the results were well correlated with their low binding affinity with FcRn, FcyRs, and complement C1q. This result was quite understandable because SHR-A1403 and SHR-A1403 mAb were originated from a humanized IgG2 antibody. The presented in vivo anti-tumor efficacy was predominantly influenced by the cytotoxicity of the conjugated toxin rather than the inhibition of c-Met signaling by the monoclonal antibody or ADCC and CDC activities; SHR-A1403 showed better anti-tumor efficacy than the unconjugated antibody, although they had equivalent inhibition of c-Met downstream effectors and similar ADCC and CDC activities due to the IgG2 subtype.

PDX models are considered to be the most predictive for evaluating potential anti-tumor therapies and have been widely used in preclinical anti-tumor drug discovery $[26,27]$. The antitumor efficacy of SHR-A1403 was further confirmed in a c-Metoverexpressing HCC PDX model, which was unresponsive to the naked antibody SHR-A1403 mAb (Fig. 3). Similar findings were obtained in an NSCLC PDX model that is refractory to ABT-700 with IHC-confirmed high c-Met expression after treatment with ABBV-399 [20]. These encouraging preclinical efficacy results and the clinical outcome with ABBV-399 will decrease the difficulty of screening compared with currently available therapies and broaden the patient population. The results also indicated that patients unresponsive or refractory to small molecule or antibody inhibitors could receive benefit from the ADC therapy.

Currently, the first-in-human study of SHR-A1403 has been initiated in the US (NCT03398720). In the dose escalation part, tolerance, safety, pharmacokinetics, and efficacy will be evaluated in patients with solid tumors; in the expansion part, efficacy will be further evaluated in solid tumor patients with high c-Met expression levels. In consideration of the preclinical pharmacokinetic properties of SHR-A1403 obtained in preclinical models and dosing frequency adopted by other ADCs [19, 28-30], a onceevery-three-weeks dosing regimen was selected for SHR-A1403 in this first-in-human study.

In summary, SHR-A1403 is a novel c-Met ADC with a favorable preclinical efficacy profile that encourages an advance into the clinical stage as a promising therapy that could overcome limitations associated with other classes of c-Met inhibitors.

\section{AUTHOR CONTRIBUTIONS}

SHG and LGL conceived the project and planned the experiments. CYY and LW designed and participated in all the experiments and wrote the paper. HTQ participated in in vitro experiments. XS and MT analyzed the results. SHG, LGL and LSZ reviewed and edited the manuscript. All authors contributed to and approved the final manuscript.

\section{REFERENCES}

1. Mo HN, Liu P, Targeting MET. in cancer therapy. Chronic Dis Transl Med. 2017;3:148-53

2. Zhang $\mathrm{Y}$, Xia M, Jin K, Wang S, Wei H, Fan C, et al. Function of the c-Met receptor tyrosine kinase in carcinogenesis and associated therapeutic opportunities. Mol Cancer. 2018;17:45-58.

3. Gow CH, Hsieh MS, Wu SG, Shih JY. A comprehensive analysis of clinical outcomes in lung cancer patients harboring a MET exon 14 skipping mutation compared to other driver mutations in an East Asian population. Lung Cancer. 2017;103:82-9.

4. Bahrami A, Shahidsales S, Khazaei M, Ghayour-Mobarhan M, Maftouh M, Hassanian SM, et al. C-Met as a potential target for the treatment of gastrointestinal cancer: Current status and future perspectives. J Cell Physiol. 2017;232:2657-73.

5. Granito A, Guidetti E, Gramantieri L. c-MET receptor tyrosine kinase as a molecular target in advanced hepatocellular carcinoma. J Hepatocell Carcinoma. 2015;2:29-38.

6. Lee D, Sung ES, Ahn JH, An S, Huh J, You WK. Development of antibody-based cMet inhibitors for targeted cancer therapy. Immunotargets Ther. 2015;4:35-44.

7. Parikh PK, Ghate MD. Recent advances in the discovery of small molecule c-Met Kinase inhibitors. Eur J Med Chem. 2018;143:1103-8.

8. Grullich C. Cabozantinib: a MET, RET, and VEGFR2 tyrosine kinase inhibitor. Recent Results Cancer Res. 2014;201:207-14.

9. Heigener DF, Reck M. Crizotinib. Recent Results Cancer Res. 2014;201:197-205.

10. Best J, Schotten C, Lohmann G, Gerken G, Dechene A. Tivantinib for the treatment of hepatocellular carcinoma. Expert Opin Pharmacother. 2017;18: 727-33.

11. Gavine PR, Ren Y, Han L, Lv J, Fan S, Zhang W, et al. Volitinib, a potent and highly selective c-Met inhibitor, effectively blocks c-Met signaling and growth in c-MET amplified gastric cancer patient-derived tumor xenograft models. Mol Oncol. 2015;9:323-33.

12. Bauer TM, Schuler M, Berardi R, Lim WT, Van Geel R, De Jonge M, et al. MINI01.03: phase (Ph) I study of the safety and efficacy of the CMET Inhibitor Capmatinib (INC280) in Patients with Advanced CMET+ NSCLC: topic: medical oncology. J Thorac Oncol. 2016;11:S257-8.

13. Pietronave S, Forte G, Locarno D, Merlin S, Zamperone A, Nicotra G, et al. Agonist monoclonal antibodies against HGF receptor protect cardiac muscle cells from apoptosis. Am J Physiol Heart Circ Physiol. 2010;298:H1155-65.

14. Jin H, Yang R, Zheng Z, Romero M, Ross J, Bou-Reslan H, et al. MetMAb, the onearmed 5D5 anti-c-Met antibody, inhibits orthotopic pancreatic tumor growth and improves survival. Cancer Res. 2008;68:4360-8.

15. Jun HT, Sun J, Rex K, Radinsky R, Kendall R, Coxon A, et al. AMG 102, a fully human anti-hepatocyte growth factor/scatter factor neutralizing antibody, enhances the efficacy of temozolomide or docetaxel in U-87 MG cells and xenografts. Clin Cancer Res. 2007;13:6735-42. 
16. Garber K. MET inhibitors start on road to recovery. Nat Rev Drug Discov. 2014;13:563-5.

17. Sheridan C. Genentech to salvage anti-MET antibody with subgroup analysis. Nat Biotechnol. 2014;32:399-400.

18. Dan N, Setua S, Kashyap VK, Khan S, Jaggi M, Yallapu MM, et al. Antibody-drug conjugates for cancer therapy: chemistry to clinical implications. Pharm (Basel). 2018;11:32-53.

19. Strickler JH, Nemunaitis JJ, Weekes CD, Ramanathan RK, Angevin E, Morgensztern D, et al. Phase 1, open-label, dose-escalation and expansion study of ABBV-399, an antibody drug conjugate (ADC) targeting c-Met, in patients (pts) with advanced solid tumors. J Clin Oncol. 2016;34:2510.

20. Wang J, Anderson MG, Oleksijew A, Vaidya KS, Boghaert ER, Tucker L, et al. ABBV399, a c-Met antibody-drug conjugate that targets both MET-amplified and c-Met-overexpressing tumors, irrespective of MET pathway dependence. Clin Cancer Res. 2017;23:992-1000.

21. Wang J, Goetsch L, Tucker L, Zhang Q, Gonzalez A, Vaidya KS, et al. Anti-c-Met monoclonal antibody ABT-700 breaks oncogene addiction in tumors with MET amplification. BMC Cancer. 2016;16:105-18.

22. Di Renzo MF, Narsimhan RP, Olivero M, Bretti S, Giordano S, Medico E, et al. Expression of the Met/HGF receptor in normal and neoplastic human tissues. Oncogene. 1991;6:1997-2003.

23. Chalouni C, Doll S. Fate of antibody-drug conjugates in cancer cells. J Exp Clin Cancer Res. 2018;37:20-31.
24. Kalim M, Chen J, Wang S, Lin C, Ullah S, Liang K, et al. Intracellular trafficking of new anticancer therapeutics: antibody-drug conjugates. Drug Des Devel Ther. 2017;11:2265-76.

25. Xu S. Internalization, trafficking, intracellular processing and actions of antibodydrug conjugates. Pharm Res. 2015;32:3577-83.

26. Lai Y, Wei X, Lin S, Qin L, Cheng L, Li P. Current status and perspectives of patient-derived xenograft models in cancer research. J Hematol Oncol. 2017;10: 106-19.

27. Williams JA. Using PDX for preclinical cancer drug discovery: the evolving field. J Clin Med. 2018;7:41-53.

28. Almhanna K, Miron ML, Wright D, Gracian AC, Hubner RA, Van Laethem JL, et al Phase II study of the antibody-drug conjugate TAK-264 (MLN0264) in patients with metastatic or recurrent adenocarcinoma of the stomach or gastroesophageal junction expressing guanylyl cyclase C. Invest New Drugs. 2017;35: 235-41.

29. Liu JF, Moore KN, Birrer MJ, Berlin S, Matulonis UA, Infante JR, et al. Phase I study of safety and pharmacokinetics of the anti-MUC16 antibody-drug conjugate DMUC5754A in patients with platinum-resistant ovarian cancer or unresectable pancreatic cancer. Ann Oncol. 2016;27:2124-30.

30. Weekes CD, Lamberts LE, Borad MJ, Voortman J, Mc Williams RR, Diamond JR, et al. Phase I study of DMOT4039A, an antibody-drug conjugate targeting mesothelin, in patients with unresectable pancreatic or platinum-resistant ovarian cancer. Mol Cancer Ther. 2016;15:439-47. 